\title{
Genomics of microbial pathogens and host-pathogen interactions
}

\section{0: Predicting protein-protein interactions from metagenomics datasets}

Tun Kyaw, Pawan Dhar

RIKEN Advanced Research Institute, 1-7-22 Suehiro-cho, Tsurumi-ku, Japan

It is now understood that even completely-sequenced genomes present only a partial snapshot of cellular inventory and processes. The bulk of processes happen at the RNA and protein interaction levels. Since interaction information of metagenome data is generally not available, people have used genome sequence to predict protein interactions and functions. However, proteins rarely act alone. They frequently assemble with other proteins to enable cellular functions. With millions of new genes identified through metagenomics studies, the task of identifying proteins, their interactions and their function, is truly enormous. We have embarked upon the mission of virtually constructing molecular networks for organisms for which genome sequence is the only available data. As a first step, we are focusing on protein-protein interactions. We hope to implement the modules in the iMetaSys workflow. The module takes input proteins as single FASTA format and outputs as simple interaction format (SIF). In addition, we hope to use network description for annotating protein functions. Although our initial results are promising, the sheer complexity of information is likely to generate false positives. To overcome that we hope to conduct functional metagenomics experiments to reconstruct a reasonably accurate picture of molecular interactions, both within and between organisms.

\section{1: Genetic polymorphisms in TLR1 are associated with susceptibility to leprosy}

${ }^{1}$ Sunny H. Wong, ${ }^{2}$ Sailesh Gochhait, ${ }^{1}$ Fredrik O. Vannberg, ${ }^{2}$ Dheeraj Malhotra, ${ }^{1}$ Anna Rautanen, ${ }^{1}$ Suchismita Roy,

${ }^{3}$ Ramasamy Pitchappan, ${ }^{2}$ Ramesh N. K. Bamezai, ${ }^{1}$ Adrian V. S. Hill

${ }^{1}$ Wellcome Trust Centre for Human Genetics, University of Oxford, Oxford OX3 7BN, United Kingdom, ${ }^{2}$ National Centre of Applied

Human Genetics, School of life Sciences, Jawaharlal Nehru University, Lok Nayak Jai Prakash Hospital, New Delhi, India,

${ }^{3}$ Centre for Excellence in Genomic Sciences, Madurai Kamaraj

University, Madurai 625021, India
Leprosy represents a significant health burden and remains endemic in several parts of the world. There is evidence that Toll-like receptor 1 (TLR1) mediates immune response to Mycobacterium leprae, the causative agent for leprosy. We hypothesize that genetic polymorphisms in the region are associated with susceptibility to leprosy. Using Sequenom ${ }^{\circledR}$ MassArray MALDI-TOF primer extension assay, we have genotyped 15 single nucleotide polymorphisms (SNPs) in the TLR 10-6-1 gene cluster in Indian samples. Strong evidence of association was obtained at a non-synonymous SNP (rs5743618) in TLR1 where the $\mathrm{G}$ allele was associated with significant protection in the case-control samples (OR $=0.41, P=0.0000056)$. Significant associations were also observed for other SNPs in the region. In conclusion, we have demonstrated that genetic polymorphisms in the TLR 10-6-1 gene cluster are strongly associated with susceptibility to leprosy, and our results suggested that TLR1 may play a crucial role in the immunity against leprosy.

022: Genome-wide transcription analysis of interaction between the human macrophage and Mycobacterium tuberculosis during concurrent drug administration by conventional and novel methods

\section{Amit Misra, Amit Kumar Singh, Awadh Bihari Yadav}

Pharmaceutics Division, Central Drug Research Institute, Chattar Manzil, MG Road Lucknow 226001, India

Virulent strains of Mycobacterium tuberculosis (MTB) induce 'alternative activation' of the host macrophage. Studies using inhalable microparticles with or without a payload of anti-tuberculosis drugs have indicated that such microparticles can rescue MTBinfected macrophages from alternative activation and induce oxidative radicals, Th1 cytokines and apoptosis. A genome-wide transcription analysis (Affymetrix HG-U133 Plus 2.0 DNA microarray) of THP-1 derived macrophages was undertaken after exposing them to infection with $10 \mathrm{MOI}$ of MTB H37Rv at 0,12 and $24 \mathrm{~h}$ post infection. Parallel groups were treated with drugs in solution, drugcontaining microparticles or blank microparticles. About 1,500 genes were differentially upregulated and about 500 genes differentially downregulated in response to various modes of treatment. Variations were also observed in the kinetics of gene expression. Cluster analysis indicated activation of several pathways related to innate immune 
response (cytokines, chemokines, receptors and ligands), apoptosis, cytoskeleton and membrane remodeling, general metabolism and general housekeeping. Some of these results were validated at the functional level, by studying enzyme activities, concentrations and time-courses of effector molecules and rates/extents of apoptosis. The results compared well with published reports on transcription response of human macrophages to MTB infection. These results permitted a novel analysis of tripartite interaction between host, pathogen and drug. Such analysis is important from an appreciation of the fact that concurrent chemotherapy is the norm in tuberculosis, but the outcome of chemotherapy is likely to be significantly affected by the contribution of the host response to both infection and drug treatment. Our data provide a basis for considering the relative contributions of the macrophage response to infection and drug treatment. These data also indicate the ability of our proposed drug delivery system to induce beneficial host responses. Finally, the data offer insight into manifestations of drug toxicity in the host cell, and its possible impact on the host response.

\section{3: Genomic plasticity of Helicobacter pylori and impact on evolution of novel virulence mechanisms}

\section{Niyaz Ahmed}

Centre for DNA Fingerprinting and Diagnostics, Hyderabad, India

Although $H$. pylori has been connected to gastric cancer, it has never been seen as a big threat to human health in the South Asian countries and particularly in India. This prompted us to look into the population structure of $H$. pylori in India. We found that it shares genetic origins as well as its trajectory of virulence genes with the western strains. A series of putative virulence factors which are also abundant in Indian isolates, were characterized by our group. These virulence factors are encoded by the genomic plasticity region genes which constitute a putative type IV secretion system believed to be acquired horizontally. Two of these virulence factors (JH940 and HP986) potentially interacted with the human immune system under in vitro conditions. The HP986 appears to be involved in Helicobacter persistence. Most persistent microbes seemingly evolve strategies to foil host responses and gain a niche. However, it seems that there is fine tuning between microbial immune evasion and maintenance of the growth fitness. For example, H. pylori both downregulates T-cell responses through the VacA mediated cell cycle arrest, and upregulates mucosal proinflammatory pathways by CagA. Surprisingly in our studies, HP986 appears to be able to perform both the immune stimulatory and immune evasion tasks single handedly.

\section{4: Global gene expression analysis of $P$. vivax parasites causing severe malaria and interaction with the host genome}

Ashis Das, Amit Subudhi, Boopati Pon Arunachalam, Vishal Saxena, Deepak Pakalapati

Birla Institute of Technology and Science, Pilani, Biological Sciences Group, Vidya Vihar, Pilani, Rajasthan, 333031, India

Plasmodium vivax long thought to be a benign parasite has recently been shown to cause severe manifestations $(1,2)$ which include cerebral malaria, hepatic failure, renal failure etc. which are associated with sequestration linked phenomena in Plasmodium falciparum infections. The present study uses global expressions analysis of $P$. vivax parasites involved in severe manifestations and attempts to correlate the data with expression analysis of the human PBMC from the same cases. Initial data shows up-regulation of 1,594 genes (PlasmoDB) using pooled severe $P$. vivax samples in comparison to non severe manifestations.

1. Kochar D, Saxena V, Singh N, Kochar S, Kumar V, Das A (2005). Plasmodium vivax malaria. Emerg Infect Dis 11(1):132134

2. Kochar DK, Pakalapati D, Kochar SK, Sirohi P, Khatri MP, Kochar A, Das A (2007). An unexpected cause of fever and seizures. Lancet 370:908

\section{5: Effect of IL1B promoter polymorphism on down regulation of gastrin through signalling intermediates NFKB and SMAD7}

${ }^{1}$ Susanta Roychoudhury, ${ }^{2}$ Meenakshi Maitra, ${ }^{1}$ Dipanjana Datta De, ${ }^{1}$ Sumana Bhattacharjya, ${ }^{3}$ Abhijit Choudhury, ${ }^{3}$ G. K. Dhali

${ }^{1}$ Molecular and Human Genetics Division, Indian Institute of Chemical Biology, 4, Raja S.C. Mullick Road, Kolkata 700032, India, ${ }^{2}$ Department of Pediatrics and Molecular Biology, UT Southwestern Medical Center, 5323 Harry Hines Boulevard, Dallas, Texas, United States of America, ${ }^{3}$ Departments of Medicine and Gastroenterology, Institute of Postgraduate Medicine and Experimental Research, 244, AJC Bose Road, Kolkata 700 020, India

Helicobacter pylori is a gastric pathogen that chronically infects the gastric mucosa of more than half of the world's population. Two polymorphisms $-511 \mathrm{C}>\mathrm{T}$ and $-31 \mathrm{C}>\mathrm{T}$ in the IL1B promoter have been suggested as potential susceptibility loci for Helicobacter pylori associated gastro duodenal diseases. Gastrin, one of the hormones that regulate gastric acid secretion, is involved in the pathogenesis of gastroduodenal ulcerations. The present study investigated the effect of IL1B promoter polymorphism on the transcriptional activity of the human gastrin promoter in a gastric epithelial cell line, AGS, and analyzed the underlying molecular mechanisms. Treatment of AGS with IL1B resulted in a 20-fold reduction in gastrin expression. Analysis revealed that IL1B represses gastrin through TAK1, TAB1 that ultimately up regulates NFkB. A $40 \%$ release of IL1B mediated gastrin repression, as a result of inhibition of NFkB translocation, further suggested the presence of NFkB independent pathway. It was observed that IL1B up regulates Smad7, which inhibits gastrin expression by sixfolds. These results were also validated invivo, where, at least a threefold lower expression of Smad7 and NFkB in H. pylori an infected individual with ulcer compared to infected asymptomatic individuals was observed. Analysis of the gastrin promoter suggests the involvement of repressor complexes consisting of NCOR and HDAC1 in IL1B mediated gastrin repression. The differential effect of IL1B promoter variants on its transcription and subsequent regulation on gastrin expression was also investigated. There was almost threefold increase in IL1B expression when AGS cells were transfected with -31TIL1B expression construct in comparison to -31 CIL1B. The -31 TIL1B induced a twofold greater repression of the gastrin luciferase activity compared to the -31 CIL1B. This signaling of the variant allele driven IL1B revealed an almost 1.5-fold greater expression NFkB in the -31TIL1B transfected AGS cells. Experiments with HDAC inhibitor also suggested that at a particular dose, the repression on the gastrin promoter in -31CIL1B-transfected cells was greater than in cells transfected with -31TIL1B. The invitro study has, therefore, successfully reflected at least part of the invivo scenario resulting from genetic polymorphisms. 